\title{
Lipoma endobronquial
}

\section{Endobronchial lipoma}

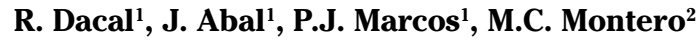

\section{RESUMEN}

El lipoma endobronquial es un tumor benigno extremadamente raro, que suele causar síntomas por obstrucción bronquial. En la tomografía computarizada de tórax se presenta como una masa homogénea de densidad grasa que no capta contraste. El diagnóstico definitivo se realiza con broncoscopia y biopsia del tumor. El tratamiento de elección es la resección por vía endoscópica. Presentamos un caso de lipoma endobronquial en una paciente de 74 años, con antecedente de carcinoma papilar de tiroides, resecado por vía endoscópica.

Palabras clave: Lipoma. Endobronquial. Benigno. Broncoscopia.

\begin{abstract}
Endobronchial lipoma is an extremely rare benign tumour, which usually causes symptoms related with bronchial obstruction. Chest computed tomography shows a homogeneous mass with fat density and without contrast enhancement. Definitive diagnosis is made with bronchoscopy and biopsy of the tumour. The treatment of choice is endoscopic resection. We present a case of an endobronchial lipoma in a 74 yearold patient, with a medical history of papilar thyroid gland carcinoma, resected endoscopically.
\end{abstract}

Key words. Lipoma. Endobronchial. Benign. Bronchoscopy.
1. Servicio de Neumología. Complexo Hospitalario Universitario. Ourense.

2. Complexo Hospitalario Universitario. A Coruña.

Recepción: 26 de junio de 2012

Aceptación provisional: 16 de julio de 2012

Aceptación definitiva: 18 de julio de 2012

\section{Correspondencia:}

Raquel Dacal Quintas

Servicio de Neumología

Complexo Hospitalario Universitario de

Ourense

C/ Ramón Puga, 22

32005-Ourense

Galicia. España

E-mail: raqueldacal@hotmail.com 


\section{INTRODUCCIÓN}

El lipoma endobronquial es un tumor benigno muy infrecuente. Suele localizarse en los bronquios principales y lobares. Aunque hay algunos casos en pacientes asintomáticos, generalmente produce clínica por obstrucción bronquial ${ }^{1-3}$. El diagnóstico definitivo se realiza mediante broncoscopia y biopsia del tumor. El tratamiento de elección es endoscópico, pero existen una serie de circunstancias en que es necesaria la cirugía.

Presentamos el caso de un lipoma endobronquial en una mujer de 74 años, con antecedente de carcinoma papilar de tiroides, resecado vía endoscópica.

\section{CASO CLÍNICO}

Mujer de 74 años, sin hábitos tóxicos, con antecedentes de tiroidectomía total más linfadenectomía por carcinoma papilar de tiroides a tratamiento con levotiroxina sódica e hipercolesterolemia en tratamiento con pravastatina. $\mathrm{La}$ paciente consultó por presentar tos de 2 meses de evolución, expectoración blanquecina, fiebre, sudoración y disnea. Inicialmente fue tratada con dos ciclos de antibióticos con mejoría de la fiebre. Posteriormente comenzó con expectoración hemoptoica, autolimitada en los días siguientes. No refería síndrome general. En la exploración física destacaba un índice de masa corporal (IMC) de 38,67 compatible con obesidad grado 2 y roncus espiratorios bilaterales. $\mathrm{La}$ analítica no mostró alteraciones y el Mantoux fue negativo.

En las pruebas de función pulmonar se objetivó una obstrucción muy leve al flujo aéreo (capacidad vital forzada [CVF] 2.570 cc $(110 \%$ del valor teórico); volumen espirado en el primer segundo [VEF1] 1.730 cc (92,7\%); FEV1/FVC 67\%; test broncodilatador negativo).

La radiografía de tórax puso de manifiesto un aumento de densidad a nivel del lóbulo inferior derecho (LID) (Figs. 1 y 2). La tomografía computarizada (TC) de tórax mostró una atelectasia sin broncograma aéreo del segmento anterior del LID y dilatación proximal del bronquio de dicho segmento, ocupado por material hipodenso compatible con densidad grasa de aspecto nodular, de 1,6 cm. (Fig. 3).
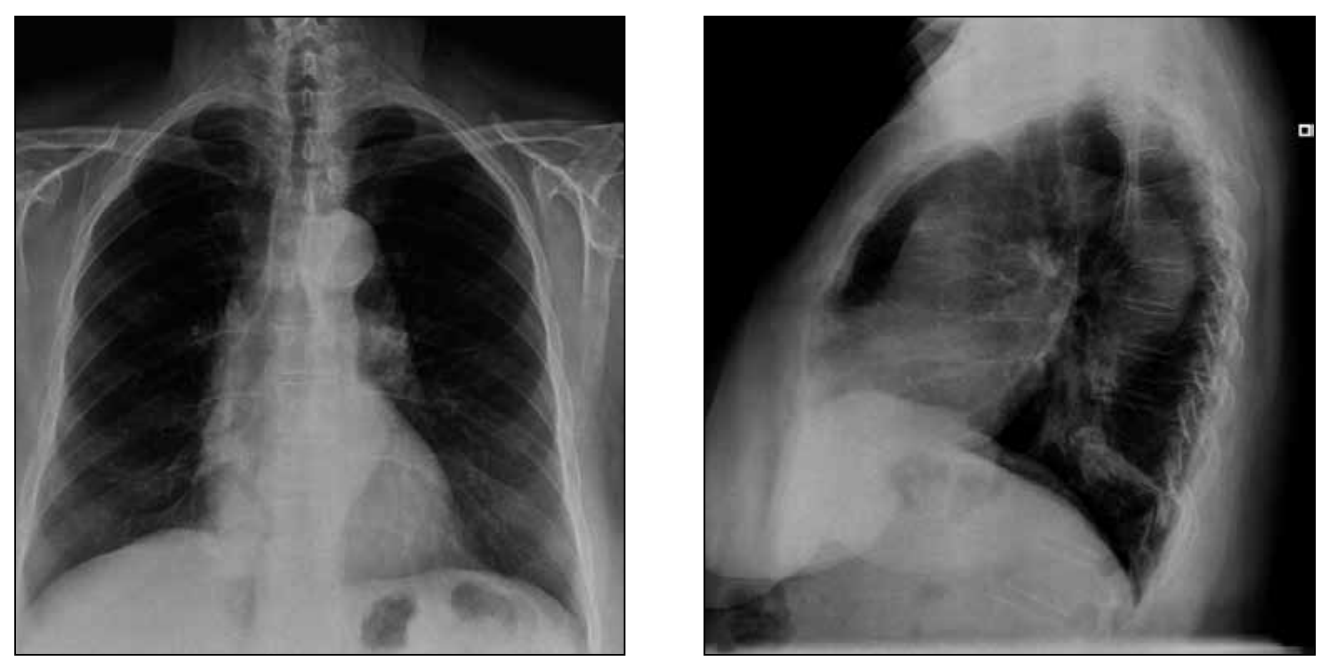

Figuras 1 y 2. Radiografía de tórax proyección póstero-anterior y lateral.

Se realizó una fibrobroncoscopia donde se observó una lesión polipoide de superficie lisa que obstruía por completo el bronquio basal an- terior del LID (Fig. 4). La biopsia bronquial mostró una proliferación bronquial de tejido adiposo maduro compatible con lipoma endobronquial. 


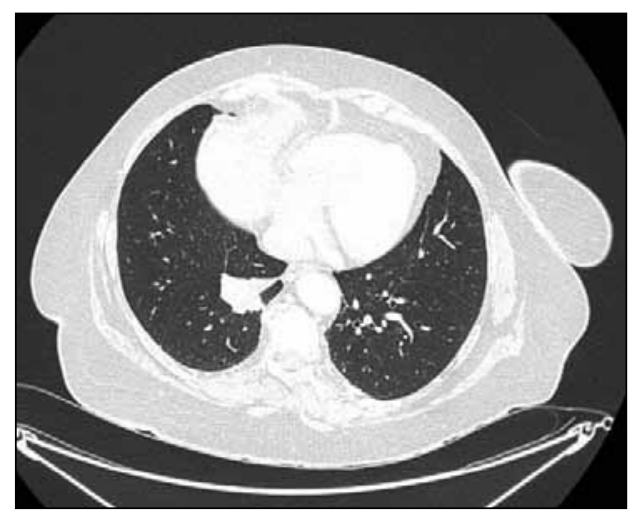

Figura 3. Corte de TC torácico donde se aprecia el lipoma.

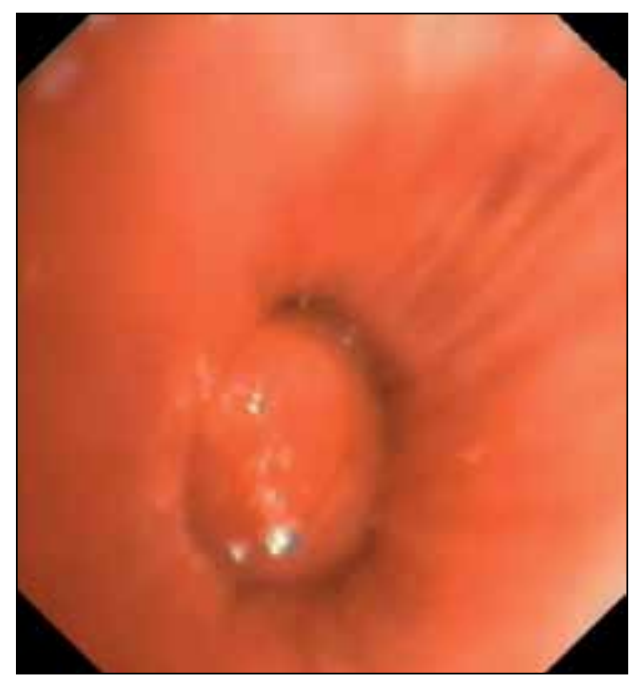

Figura 4. Imagen de fibrobroncoscopia del lipoma.

Con el diagnóstico de lipoma endobronquial se decidió tratamiento endoscópico mediante resección láser. Se procedió a fotocoagulación superficial con láser de la lesión. Se realizó un intento infructuoso de extracción con asa de diatermia por dificultad para ver la parte distal de la lesión, extrayéndose sólo una parte de la lesión, que se envió para análisis anatomopatológico, quedando el resto de la tumoración fotocoagulada de forma superficial. Un mes después, se realizó un segundo procedimiento, siendo extraído todo el material restante. No se objetivaron complicaciones tras los procedimientos.

\section{DISCUSIÓN}

Los lipomas endobronquiales son tumores benignos extremadamente raros con una incidencia de $0,1-0,5 \%$ de todos los tumores de pulmón y hasta un $13 \%$ de los tumores benignos. Tienen su origen en el tejido adiposo de la capa submucosa bronquial. Generalmente son pedunculados con tallo estrecho y cubiertos por mucosa normal, ocasionalmente pueden ser de morfología sésil. Pueden medir de 1 a $7 \mathrm{~cm} \mathrm{o}$ incluso más, y suelen localizarse en las tres primeras divisiones del árbol traqueobronquial. Predominan en hombres en la $5^{\text {a }}-6^{\text {a }}$ década de la vida y se consideran la obesidad y el hábito tabáquico como factores de riesgo para su desarrollo ${ }^{1-3}$. En nuestro caso se trata de una mujer no fumadora, pero sí obesa.

La clínica, al igual que en el caso presentado, se debe fundamentalmente a la obstrucción bronquial originada por el tumor, provocando tos, que es el síntoma más frecuente, disnea, hemoptisis, dolor torácico y neumonías de repetición. En ocasiones pueden simular asma o enfermedad pulmonar obstructiva crónica (EPOC) lo que puede retrasar el diagnóstico años o bien simular neoplasias pulmonares malignas $^{3-7}$. En algunas series se describen hasta un $25 \%$ de pacientes asintomáticos ${ }^{1}$.

En la radiografía de tórax puede haber alteraciones hasta en un $80 \%$ de $\operatorname{casos}^{1}$ tal como le sucedió a nuestra paciente, pero dichas alteraciones son indistinguibles de otros procesos (neumonía, neoplasias, etc.). En la TC el hallazgo de una masa de tejido blando con bajos valores de atenuación y que no capta contraste se considera diagnóstico. La broncoscopia y la biopsia permiten el diagnóstico definitivo en la mayoría de casos. Histológicamente, el lipoma endobronquial está formado por lóbulos de tejido adiposo separados por septos fibrosos.

El diagnóstico diferencial debe hacerse con otras lesiones endobronquiales que contengan tejido adiposo, incluyendo, tumores hamartoma-like, timolipoma, angiomiolipoma, liposarcoma, leiomioma, y teratoma, entre otros. Estos tumores, además 
de tejido adiposo, contienen estructuras con tejido blando y calcificaciones ${ }^{8}$.

El tratamiento de elección es endoscópico, mediante láser o crioterapia, pero hay una serie de circunstancias en las que se precisa de la cirugía, como son la dificultad para confirmar el diagnóstico, la destrucción del parénquima pulmonar por neumonías recurrentes, crecimiento extrabronquial, afectación subpleural o dificultad técnica endoscópica ${ }^{1}$. En nuestro caso se realizó resección mediante láser, aunque fueron precisos dos procedimientos dada la dificultad técnica para observar la parte distal a la tumoración.

Es importante realizar un diagnóstico temprano para intentar evitar la destrucción del parénquima pulmonar distal al tumor y poder aplicar un tratamiento, por vía endoscópica y evitar la cirugía extensa ${ }^{2,9}$.

\section{BIBLIOGRAFÍA}

1. Muraoka M, Oka T, Akamine S, Nagayasu T, IseKI M, SuYAma N et al. Endobronchial lipoma. Review of 64 cases reported in Japan. Chest 2003; 123: 293-296.

2. URibe-Etxebarría N, Lorenzo M, Jiménez U, CALvo I, Rumbero JC, Rojo R, PAC J. Lipoma endobronquial. Consideraciones terapéuticas. Cir Esp 2006; 80: 109-110.

3. Schraufnagel DE, Morin JE, Wang NS. Endobronchial lipoma. Chest 1979; 75: 97-99.

4. Huisman C, Van Kralinger KW, Postmus PE, SuTEDJA TG. Endobronchial lipoma: a series of three cases and the role of electrocautery. Respiration 2000; 67: 689-692.
5. MAGRo CM, Ross P. Endobronchial mimics of primary endobronchial carcinoma: A clinical study of 25 cases. Can Resp J 2005; 12: 123127.

6. Irani F, Kumar B, Reddy P, Narwal-Chadha R, KasMANI R, TITA J. An endobronchial lipoma mimicking asthma and malignancy. Prim Care Respir J 2010; 19: 281-283.

7. Casanova Espinosa A, Cisneros Serrano C, Girón Moreno RM, Oliver MJ, Moreno Balsalobre R, ZAMORA GARCÍA E. Pleural empyema associated with endobronchial lipoma. Arch Bronconeumol 2005; 41: 172-174.

8. OPOKA L, LEWANDOWSKA K, LANGFORT R, RUDZIŃSKI P. Recurrence of endobronchial lipoma. Pneumonol Alergol Pol 2010; 78: 356-361.

9. Iwabuchi H, Kamura T, Tanaka M, Kato H. A case of endobronchial lipoma. Diagn Ther Endosc 1999; 5: 263-267.

10. Ouadnouni Y, Bouchikh M, BeKarsabein S, Achir A, Smahi M, Msougar Y et al. Endobronchial lipoma a rare cause of pleural empyema: a case report. Cases J 2009; 2: 6377.

11. Basoglu A, Celik B, Akdag AO, Sengul AT. Endobronchial lipoma: a rare cause of bronchial occlusion. Interact CardioVasc Thorac Surg 2004; 3: 263-264.

12. Cockcroft DW, Copland GM, Donevan RE, GourLAY RH. Endobronchial lipoma: two cases and review of the literature. CMA Journal 1976; $115 ; 326-328$.

13. Gaerte SC, Meyer CA, Winer-Muram HT, Tarver RD, Conces DJ. Fat-containing lesions of the chest. RadioGraphics 2002; 22: S61-S78.

14. Matsuba K, Saito T, Ando K, Shirakusa T. Atypical lipoma of the lung. Thorax 1991; 46: 685.

15. YoKozaKi M, Kodarna T, YoKose T, Nishimura M, Yoshida J, Mizokami H, NAGaI K. Endobronchial lipoma: A report of three cases. Jpn J Clin Oncol 1996; 26: 53-57. 